
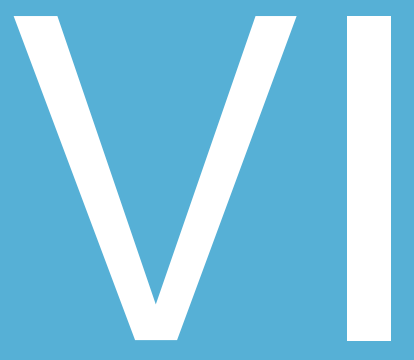

\title{
PERFIL EDUCATIVO PERUANO 2010
}

\section{Peruvian Educational Profile 2010}

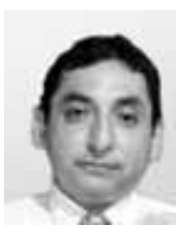

\section{Alexander De La Cruz}

Licenciado en Administración por la Universidad Peruana Unión. Magíster en Administración por la Universidad Nacional del Altiplano. Docente Universitario de la Facultad de Ciencias Empresariales. Actualmente se desempeña como Gerente de la Universidad Peruana Unión sede Tarapoto.

\section{Hernán Mendoza}

Alumno de la carrera de Marketing y Negocios Internacionales de la Universidad Peruana Unión. Fundador y líder del grupo de Investigación de Mercado "Target" de la Universidad Peruana Unión. 


\section{Resumen}

Esta investigación busca identificar las preferencias que manifiestan los estudiantes por estudiar en una determinada institución universitaria, Por otra parte, se pretende medir el interés de los alumnos por educarse en nuevas carreras profesionales y técnicas, factibles a ser ofrecidas por la Universidad Peruana Unión. En relación a los objetivos, se aplicó un cuestionario directo a la totalidad de los alumnos que están por egresar de cuarto y quinto año de secundaria, haciendo un total de 555 jóvenes. Entre los resultados se encontraró que $61.4 \%$ de jóvenes prefieren las universidades privadas. De la muestra total de 555 estudiantes adventistas 27.5\% de estos les gustaría estudiar en la Universidad Peruana Unión. Los resultados de estas pruebas fueron analizados a través del paquete de análisis estadístico para la investigación educativa Visual Basic, mediante el análisis de frecuencias absolutas y porcentuales, media y moda. Los jóvenes de los colegios adventistas del departamento de San Martín tienen preferencia por las universidades privadas.

Palabras claves: Posicionamiento, demanda estudiantil, investigación de mercados, carreras universitarias.

\section{Abstract}

This research seeks to identify preferences expressed by students studying at a particular university, by the same way is to measure the students' interest in exploring new professional and technical careers, feasible to be offered by the Universidad Peruana Unión. In relation to the objectives, a questionnaire was applied directly to all the students who are graduating from fourth and fifth grade, making a whole of 555 young people. The results could be found that $61.4 \%$ of young people prefer private universities. From the whole sample of 555 , the $27.5 \%$ are Adventist students and they would like to study at the Universidad Peruana Union. The results of these tests were analyzed through statistical analysis package in Visual Basic educational research, through analysis of absolute frequencies and percentages, mean and mode. Young people from schools Adventists Department of San Martin have a preference for private universities.

Keywords: Positioning, student demand, market research university 


\section{Introducción}

Elegir la educación universitaria para los hijos implica adecuar una equilibrada relación entre la demanda estudianti la oferta de carreras profesionales y los recursos que disponen las insti s. Todo ello sin que con esto se pretenda negar que las insti de educación superior tengan, entre sus propósitos, la responsabilidad de ser promotoras de cambio de estructuras sociales rígidas, lo cual implica la oferta de carreras cuyo objeti o sea "abrir la brecha" dentro de los sistemas sociales anquilosados por la tradición o la apatí por el progreso.

Para planear una educación equilibrada y poder ofrecer las carreras profesionales que se necesita, hay que hacer uso de la investi ación de mercados y así entender las condiciones del mercado y poder nivelar el centro de estudio a las exigencias de la demanda. Esto permiti á:

Promover una demanda racional de educación superior orientada con base a necesidades reales del país y genuinos intereses vocacionales.

Ampliar la cobertura de la demanda social de educación superior con una mayor y más adecuada oferta educati a por parte del sistema, en función de las necesidades de la sociedad y los recursos disponibles.

En conveniencia con las oportunidades que nos brinda la investi ación de mercados, la Universidad Peruana Unión (en adelante UPeU), mediante su grupo de investi ación Target (Centro de Aplicación de la Escuela de Marketi y Negocios Internacionales de la UPeU, Tarapoto); realizó una investi ación de mercado, con el propósito de explorar la demanda de educación universitaria y conocer las tendencias hacia nuevas carreras profesionales.

Algunos investi adores como Piscoya (2006), y Centro de Investigación y Documentación Educati a [CADE] (1994), realizaron estudios similares sobre la demanda de la educación universitaria, llegando a establecer patrones que son fuentes de consulta hoy.

El objeti o de esta investi ación es identifi ar las preferencias que manifi ten los estudiantes por estudiar en una determinada insti educati a, del mismo modo se pretende medir el interés de los alumnos por estudiar nuevas carreras profesionales y técnicas, facti s de ser ofrecidas por la Universidad Peruana Unión.

Asimismo, conocer el posicionamiento de la Universidad Peruana Unión y la preferencia que manifi ten los padres por enviar a sus hijos a 
una insti educati a y el proceso de elección, con el objeto de saber si las actuales carreras que ofrece responden a una necesidad regional.

\section{Materiales y métodos}

Según Malhotra (2008, p.7), la investigación de mercados es la función que conecta al consumidor, al cliente y al público con el vendedor mediante la información, la cual se utiliza para identificar y definir las oportunidades y los problemas del marketing; para generar, perfeccionar y evaluar las acciones que ello involucra; asimismo, para monitorear su desempeño y mejorar su comprensión como un proceso.

La investigación de mercados especifica la información que se requiere para analizar esos temas, diseña las técnicas para recabar la información, dirige y aplica el proceso de recopilación de datos, analiza los resultados y comunica los hallazgos y sus implicaciones.

La investigación de mercados consiste en la identificación, recopilación, análisis, difusión y uso sistemático y objetivo de la información con el propósito de mejorar la toma de decisiones relacionadas con la identificación y marketing (Malhotra, 2008, p. 8). Además Zikmund y Babin(2008), confirman que la investigación de mercados es la aplicación del método científico en la búsqueda de la verdad acerca de los fenómenos de marketing. Estas actividades incluyen la definición de oportunidades y problemas del marketing, la generación y evaluación de ideas, monitoreo del desempeño y la comprensión del proceso del marketing. Dicha investigación es más que la mera aplicación de encuestas. Este proceso incluye el desarrollo de ideas, teorías, la definición del problema, la búsqueda y acopio de información, el análisis de datos, y la comunicación de las conclusiones y consecuencias.

Esta investigación de mercado se aplicó en el departamento de San Martín y fue delimitado a las instituciones educativas adventistas, según el orden que sigue a continuación; Tarapoto: Colegio José de San Martín, Moyobamba: ASEANOR MOYOBAMBA, Rioja: ASEANOR ALTOMAYO, Nueva Cajamarca: ASEANOR NUEVA CAJAMARCA, Tocache: FERNANDO STHAL.

La información teórica se obtuvo de revistas y libros que se encuentran en la biblioteca universitaria de la UPeU, Tarapoto. La búsqueda incluyó también consultas en línea a través del sistema EBSCO (Biblioteca y base de datos virtual) que opera en la Universidad Peruana Unión.

En relación con los objetivos, se aplicó un cuestionario directo a la totalidad de los alumnos que están por egresar de 4 to y 5 to año. 
Para validar el instrumento se solicitó el juicio de expertos, siendo validado por el ingeniero Edison Effer Apaza, docente y especialista en la cátedra de Estadística, así como por el magíster Alexander De La Cruz, especialista en Mercadología, quienes hicieron la validación del instrumento para el tema de estudio. Estos estudiosos también evaluaron la claridad, concisión y relevancia de los enunciados.

\section{Prueba piloto}

Para la validación estadística se realizó una prueba piloto en la UPeU, Tarapoto, previa la recolección de datos con el fin de obtener información que ayude a mejorar la validez y confiabilidad del instrumento y detectar problemas en la validez de construcción, de contenido y de criterio. Sobre la forma de administración y calificación de la escala recomendados por autores en investigación (Hernández, 2003), se aplicó el instrumento a 45 alumnos en Tarapoto, con características similares a las del objeto de estudio. Dicha prueba y su respectivo análisis ayudó a mejorar el formato, la redacción, la claridad y la concisión de los ítems que integran el instrumento.

Posteriormente se procedió a la validación estadística del instrumento mediante el análisis de la fiabilidad de ítem por ítem del test, así como la validez y coeficiente de confiabilidad del instrumento mediante el alfa de Cronbach, que midió la consistencia interna arrojando un coeficiente total de 0.77 , que es valorado como alto o consistente. El instrumento quedó expedito para ser aplicado a la muestra basada en su alta validez y confiabilidad.

Para fi de esta investi ación es necesario entender algunos términos.

\section{Posicionamiento}

Lamb y otros (2006 p. 249), argumentan: "La posición es el lugar que ocupa un producto, marca o grupo de productos en la mente de los consumidores en relación con las ofertas de la competencia".

\section{Servicio}

Se refiere a un hecho, un desempeño o un esfuerzo que no es posible poseer físicamente. 


\section{Demanda estudiantil}

Hamquist (citado por Salinas 2006 p. 87), propone la demanda de la educación que surge de los deseos y aspiraciones de los individuos, en contraposición con la demanda educati a, basada en las necesidades de personas de la sociedad. Sin embargo, el concepto de demanda social sería aplicable principalmente en educación superior, donde existe una situación de opción, por mucho que esta sea medida por múlti elementos, siendo obligatoria la educación básica, la elección se reduce a una disti entre la oferta pública y la privada.

\section{Resultados y discusión}

TABLA 1: Descriptivos de preferencias según el centro superior

\begin{tabular}{|c|c|c|}
\hline & Frecuencia & Porcentaje \\
\hline Universidad Particular & 341 & 61.4 \\
\hline Universidad Estatal & 179 & 32.3 \\
\hline Instituto Particular & 19 & 3.4 \\
\hline Instituto Estatal & 6 & 1.1 \\
\hline Otro & 10 & 1.8 \\
\hline Total & 555 & 100 \\
\hline
\end{tabular}

Fuente: Centro de Aplicación Target

TABLA 2: Descriptivos de elección de la carrera

$\begin{array}{lcc} & \text { Frecuencia } & \text { Porcentaje } \\ \text { Padres } & 220 & 39.6 \\ \text { Profesores } & 4 & .7 \\ \text { Familiares } & 41 & 7.4 \\ \text { Amigos } & 7 & 1.3 \\ \text { Hermanos } & 6 & 1.1 \\ \text { Por mi cuenta } & 268 & 48.3 \\ \text { Otro } & 9 & 1.6 \\ \text { Total } & 555 & 100.0\end{array}$

Fuente: Centro de Aplicación Target 
- APUNTES AÑOI • NÚMERO 1 • ISSN: 2225 - 7136

Tabla 3: Tabla preferencia universitaria

\begin{tabular}{|c|c|c|}
\hline UNIVERSIDADES & 1ra Opción & Porcentaje \\
\hline U. PERUANA UNIÓN & 149 & 28 \\
\hline PONTIFICIA UNIVERSIDAD CATÓLICA DEL PERÚ & 66 & 12 \\
\hline U. CÉSAR VALLEJO & 65 & 12 \\
\hline U. ALAS PERUANAS & 44 & 8 \\
\hline U. DE SAN MARTÍN DE PORRES & 29 & 5 \\
\hline U. PERUANA DE CIENCIAS APLICADAS & 27 & 5 \\
\hline U. RICARDO PALMA & 22 & 4 \\
\hline U. DE HUÁNUCO & 21 & 4 \\
\hline U. ADVENTISTA DE MONTEMORELOS & 13 & 2 \\
\hline U. PRIVADA DE PIURA & 12 & 2 \\
\hline U. PRIVADA DEL NORTE & 10 & 2 \\
\hline U. SEÑOR DE SIPÁN & 10 & 2 \\
\hline U. TECNOLÓGICA DEL PERÚ & 9 & 2 \\
\hline U. CIENTÍFICA DEL PERÚ & 8 & 1 \\
\hline U. DEL PACÍFICO & 7 & 1 \\
\hline U. DE LIMA & 6 & 1 \\
\hline U. PRIVADA ANTENOR ORREGO & 6 & 1 \\
\hline U. ADVENTISTA DEL PLATA & 5 & 1 \\
\hline U. LOS ÁNGELES DE CHIMBOTE & 4 & 1 \\
\hline U. PERUANA CAYETANO HEREDIA & 4 & 1 \\
\hline U. PERUANA UNIÓN - LIMA & 4 & 1 \\
\hline
\end{tabular}


Alexander De La Cruz - Hernán Mendoza

\begin{tabular}{lcc|}
\hline U. SAN IGNACIO DE LOYOLA & 4 & 1 \\
\hline U. PRIVADA DE PUCALLPA & 3 & 1 \\
\hline ESAN & 2 & 0 \\
U. INCA GARCILASO DE LA VEGA & 2 & 0 \\
\hline U. PRIVADA ANTONIO GUILLERMO URRELO & 2 & 0 \\
\hline U. DE CHICLAYO & 2 & 0 \\
\hline U. ADVENTISTA, USA & 1 & 0 \\
\hline U. PRIVADA DE TUMBES & 1 & 0 \\
\hline U. PRIVADA DEL ORIENTE & 1 & 0 \\
\hline OTRAS RESPUESTAS VÁLIDAS & 1 & 0 \\
\hline \multicolumn{1}{c}{ TOTAL } & 539 & 100 \\
\hline
\end{tabular}

Fuente: Centro de Aplicación Target

Tabla 4: Descriptivos de elección universitaria

\begin{tabular}{|lcc|} 
& Frecuencia & Porcentaje \\
\hline MEJOR NIVEL ACADÉMICO & 241 & 45.1 \\
\hline NO HUELGAS & 135 & 25.3 \\
\hline PREFERENCIA & 53 & 9.9 \\
\hline FÁCIL INGRESO & 49 & 9.2 \\
\hline BENEFICIOS & 35 & 6.6 \\
\hline NO ESTÁ INTERESADO & 13 & 2.4 \\
\hline PREFIEREN MIS PADRES & 8 & 1.5 \\
\hline \multicolumn{1}{|c|}{ TOTAL } & 534 & 100 \\
\hline
\end{tabular}

Fuente: Centro de Aplicación Target 


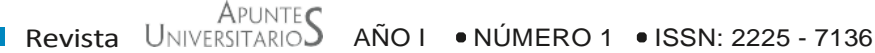

Cuadro 1: Carreras de mayor demanda

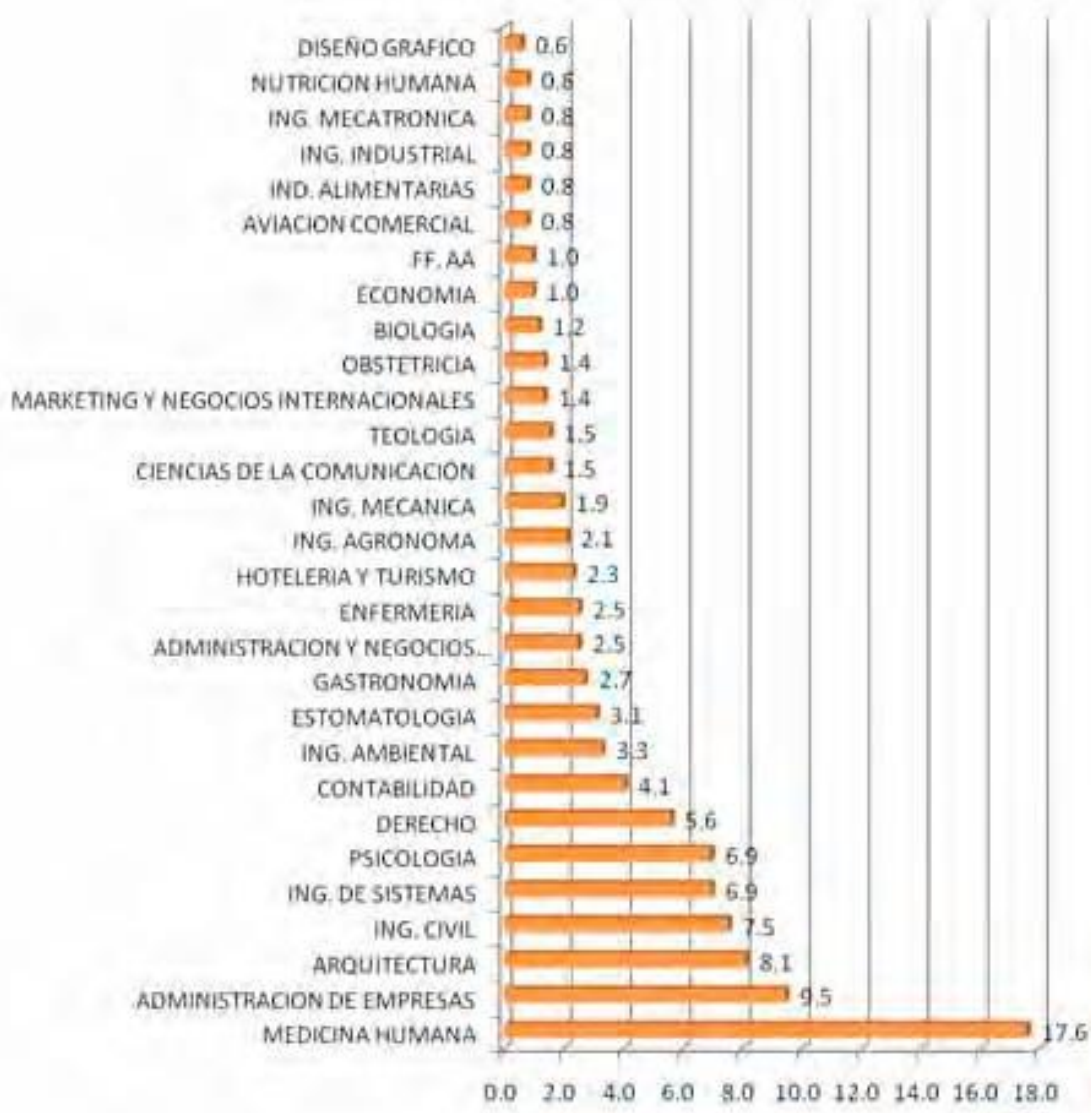

Como se puede observar en la tabla 1, representando el $61.4 \%, 341$ prefieren las universidades privadas; 179 (32.3\%), las universidades estatales; mientras que los 35 restantes (6.3\%), prefieren los institutos.

Según la tabla 2, 268 alumnos, que representan el 48.3\%, han decidido estudiar por cuenta propia; mientras que un 39.6\%, que suman 220 estudiantes, son influenciados por la decisión de sus padres. 
Según el tabla 3, 149 estudiantes, que representan el 27.5\% alumnos, les gustaría estudiar en la Universidad Peruana Unión; a 66 estudiantes, en la Pontificia Universidad Católica del Perú, que representa un $12.2 \%$; seguido de 65 estudiantes, que representan el 12\%, desean estudiar la carrera de su preferencia en la Universidad César Vallejo.

En la tabla 4, observamos que la razón primordial por la que eligen estudiar en una universidad privada es por el mejor nivel académico, representando por un 45.1\%; seguido por la razón que no hay huelgas con un $25.3 \%$.

Cuadro 1, de un total de 555 estudiantes de colegios adventistas encuestados, se obtuvo la siguiente información con respecto a la preferencia en segunda instancia de una carrera universitaria o técnica: el $8 \%$ prefiere la carrera profesional Medicina Humana; seguido de un 6.8\%, que tiene preferencia por Administración de Empresas; como tercera opción, con un $6.4 \%$, la carrera de Arquitectura; en cuarto lugar, con un 6.4\%, la carrera profesional de Ingeniería de Sistemas. La lista continúa con carreras profesionales tales como: Psicología, Contabilidad, Derecho e Ingeniería Ambiental, con porcentajes similares.

\section{Conclusiones}

1. Los jóvenes de los colegios adventistas del departamento de San Martín tienen preferencia por las universidades privadas.

2. Para la elección de la carrera universitaria la influencia de los padres no es muy elevada; sin embargo, podemos observar y analizar que es un factor muy importante para que el joven elija la carrera a estudiar.

3. En las instituciones adventistas del departamento de San Martín, se puede apreciar que la Universidad Peruana Unión está posicionada en la mente de los jóvenes. Lo cual ayuda a cumplir con los objetivos y de esta manera emplear estrategias para el crecimiento y posicionamiento de la Universidad Peruana Unión.

4. La Universidad Peruana Unión cuenta con múltiples ventajas que alienta al joven estudiante a cumplir sus metas profesionales. Es por ello que la razón primordial por la que eligen una universidad privada es por la calidad de servicio que esta brinda.

5. En el caso de la Universidad Peruana Unión, filial Tarapoto, se eligió las carreras de Administración de Empresas e Ingeniera Ambiental, ya que ambas responden a los intereses de los jóvenes encuestados. 


\section{Recomendaciones}

1. Es conveniente realizar nuevas investigaciones para medir el posicionamiento de la UPeU, Tarapoto y las nuevas demandas de nuestro público objetivo.

2. Debemos tener en cuenta los hallazgos de esta investigación para realizar estrategias en la captación de nuevos alumnos.

3. Se recomienda considerar en relación al tema de estudios si el joven es financiado por sus padres, se autosostiene o recibe ayuda de un tercero.

4. Se debe hacer más énfasis en la promoción y publicidad de la universidad, dando a conocer las carreras y beneficios que ofrece la institución y difundirlas en medios masivos, para que tanto los jóvenes adventistas como no adventistas puedan obtener información detallada.

5. Aprovechar las redes sociales para una mayor difusión de los servicios de la Universidad Peruana Unión.

6. Mediante esta investigación se recomienda ampliar las carreras universitarias, teniendo en cuenta los resultados obtenidos en la región.

Alexander De La Cruz

Universidad Peruana Unión

e-mail: advstar_1@hotmail.com

Hernán Mendoza

Universidad Peruana Unión e-mail: hernanmendoza12@hotmail.com

Recibido: 1 de septiembre de 2011 Aceptado: 12 de octubre de 2011 


\section{Alexander De La Cruz - Hernán Mendoza}

\section{Referencias}

Bernal, C. (2006). Metodología de la investigación para Administración, Economía, Humanidades y Ciencias Sociales. $2^{\text {a }}$ ed. México: Pearson Educación, 304 p. ISBN 970-26-0645-4.

Centro de Investigación y Documentación (CADE), (1995). Doce años de investigación educativa: Catálogo, 1983-1994. Madrid: Centro de Publicaciones del Ministerio de Educación y Ciencia CIDE, 355 p. ISBN: 84-209-2701-X

Hernández, R., Fernández, C., Baptista, L. (2003). Metodología de la Investigación. $3^{\text {a }}$ ed. México: Mc Graw Hill. 500 p.

Lamb, C., Hair, J., Mc Daniel, C. (2006). Marketi $\quad$ 8a ed. México: Thomson. 746 p. ISBN 970-686-547-0

Malhotra, N. (2008). Investigación de mercados. 5a Ed. México: Pearson educación.920 p. ISBN 978-970-26-1185-1.

Piscoya, L., et al (2006) Ranking universitario en el Perú. Lima, 195 p.

Salinas, S. (2006). Demanda educativa de la población jornalera agrícola migrante: estadística y concepto. México: CGIB. 87p. ISBN: 9789708141505

Zikmund, C. y Babin, B. (2008) Investigación de mercados. México: Cengage Learning. 746 p. 